\title{
Extension de l'aire de distribution connue de la Musaraigne fuligineuse, Sorex fumeus, dans le nord-est du Québec
}

\author{
JeAn-François Desroches ${ }^{1}$ et IsABelle PiCARD \\ ${ }^{1}$ Collège de Sherbrooke, Département des Techniques d'écologie appliquée, 475 du Parc, Sherbrooke, Québec J1K 4K1 \\ Canada
}

Desroches, Jean-François, et Isabelle Picard. 2004. Extension de l'aire de distribution connue de la Musaraigne fuligineuse, Sorex fumeus, dans le nord-est du Québec. Canadian Field-Naturalist 118(3) : 441-442.

La Musaraigne fuligineuse (Sorex fumeus) figure sur la liste des espèces de la faune vertébrée susceptibles d'être désignées menacées ou vulnérables au Québec. Le 7 juin 1999, nous avons récolté un individu mort en bordure de la rivière Saint-Nicolas, à Godbout, dans la région de la Côte Nord. Cette observation constitue une extension de l'aire de répartition connue de l'espèce d'environ $200 \mathrm{~km}$ vers le nord-est.

Mots-clés : Musaraigne fuligineuse, Sorex fumeus, aire de distribution, Québec.

La Musaraigne fuligineuse (Sorex fumeus) est présente dans le nord-est de l'Amérique du Nord, des Great Smokies des Carolines au sud jusqu'au centre de l'Ontario et aux Maritimes vers le nord (Banfield 1977). Au Québec, la mention la plus nordique de l'espèce se situe à Val-Jalbert, aux abords du lac SaintJean (van Zyll de Jong 1983). Sa répartition connue vers le nord-est, sur la rive nord du fleuve Saint-Laurent, ne dépasse guère la rivière Saguenay (van Zyll de Jong 1983; Banfield 1977). Cette musaraigne figure sur la liste des espèces de vertébrés susceptibles d'être désignées menacées ou vulnérables au Québec (Beaulieu 1992). Les chercheurs contactés en 1989 n'avaient pas rapporté de capture récente au Québec (Beaulieu 1992) mais depuis, elle a été capturée à divers endroits notamment dans le sud de la province (G. Lupien, communication personnelle).

Le 7 juin 1999, nous avons trouvé un spécimen de la Musaraigne fuligineuse mort, probablement noyé, en bordure de la rivière Saint-Nicolas, à environ 100 mètres au sud de la route 138 à Godbout $\left(49^{\circ} 19^{\prime} 10^{\prime \prime} \mathrm{N}\right.$, $67^{\circ} 47^{\prime} 24^{\prime \prime}$ ), dans la région de la Côte Nord. L'habitat à cet endroit est une aulnaie, bordée par une sapinière à Bouleau blanc. Aucun autre micromammifère n'y a été observé. Le spécimen de Musaraigne fuligineuse a été récolté pour identification. Les mesures ont été prises sur le spécimen à l'aide d'un vernier à coulisse, par J-F. D. et S. Houde, et elles correspondent à celles de la Musaraigne fuligineuse (tableau 1). Au Québec, la Musaraigne fuligineuse peut être confondue avec la Musaraigne cendrée (Sorex cinereus), ayant toutes deux de quatre à cinq dents unicuspides visibles, la limite postérieure du foramen infraorbitaire antérieure ou près de la limite entre les molaires 1 et 2 , le trou mentonnier situé à la hauteur du protoconide de la molaire 1, et la mandibule dépourvue de foramen postmandibulaire (Maisonneuve et al. 1997). Elle s'en distingue toutefois par certaines mesures du crâne et du corps (tableau 1). Le spécimen a malheureusement été perdu quelques jours plus tard, jeté par erreur avec d'autres micromammifères morts, mais les mesures prises confirment son identification.

Cette observation constitue une extension d'aire de l'espèce de $200 \mathrm{~km}$ vers le nord-est, soit à partir de l'embouchure de la rivière Saguenay (van Zyll de Jong 1983). La Musaraigne cendrée (Sorex cinereus), une espèce plus commune au Québec (observation personnelle), a une distribution qui s'étend bien plus au

TABleau 1. Mesures comparées du spécimen de musaraigne trouvé sur la Côte Nord, de la Musaraigne fuligineuse et de la Musaraigne cendrée.

\begin{tabular}{lcccc}
\hline \hline Espèce & $\begin{array}{c}\text { Longueur } \\
\text { du crâne }(\mathrm{mm})\end{array}$ & $\begin{array}{c}\text { Largeur du } \\
\text { maxillaire }(\mathrm{mm})\end{array}$ & $\begin{array}{c}\text { Longueur } \\
\text { totale }(\mathrm{mm})\end{array}$ & $\begin{array}{c}\text { Longueur de } \\
\text { la queue }(\mathrm{mm})\end{array}$ \\
\hline Spécimen de la Côte Nord & 17,7 & 4,8 & 111,4 & 47,8 \\
Musaraigne fuligineuse & 17,5 à $18,8^{2}$ & 4,8 à $5,4^{2}$ & 110 à $127^{1}$ & 40 à $52^{1}$ \\
Musaraigne cendrée & $>17,5^{3}$ & $>4,6^{3}$ & 104 à $125^{2}$ & 42 à $54^{2}$ \\
& 15,2 à $16,9^{2}$ & 3,8 à $4,3^{2}$ & 92 à $110^{1}$ & 37 à $46^{1}$ \\
& $<17^{3}$ & $<4,6^{3}$ & 75 à $125^{2}$ & 28 à $50^{2}$ \\
\hline \hline
\end{tabular}

\footnotetext{
${ }^{1}$ Banfield 1977

${ }^{2}$ van Zyll de Jong 1983

${ }^{3}$ Maisonneuve et al. 1997
} 
nord (van Zyll de Jong 1983; Banfield 1977). La découverte de la Musaraigne fuligineuse à Godbout indique que la distribution de certaines espèces de petits mammifères est encore mal connue. En ce sens, il est permis de croire que de futures découvertes seront faites et permettront de mieux connaître les habitats fréquentés par ces espèces et de préciser leur aire de distribution dans le nord de la province. Il est à noter que cette mention de la Musaraigne fuligineuse figure dans l'Atlas des micromammifères du Québec, sans documentation publié en 2002 (Desrosiers et al. 2002).

\section{Remerciements}

Nous tenons à remercier Mme Stéphanie Houde, technicienne de la faune, pour la vérification de l'identification du spécimen de même que M. Gilles Lupien, technicien de la faune à la Société de la Faune et des Parcs du Québec, pour ses commentaires sur les captures de l'espèce au Québec. Nous remercions également le comité ZIP de l'estuaire du Saint-Laurent, qui a permis la réalisation de l'inventaire duquel la présente découverte de la Musaraigne fuligineuse a été faite.

\section{Littérature citée}

Banfield, A. W. F. 1977. Les Mammifères du Canada. Les Presses de l'Université Laval et University of Toronto Press. Publié par le Musée national des Sciences naturelles, Musées nationaux du Canada. Ottawa, Canada. 406 pages.

Beaulieu, H. 1992. Liste des espèces de la faune vertébrée susceptibles d'être désignées menacées ou vulnérables au Québec. Ministère du Loisir, de la Chasse et de la Pêche. Québec. 107 pages.

Desrosiers, N., R. Morin, et J. Jutras. 2002. Atlas des micromammifères du Québec. Société de la faune et des parcs du Québec et Fondation de la Faune du Québec. 92 pages.

Maisonneuve, C., R. Mc Nicoll, S. St-Onge, et A. Desrosiers. 1997. Clé d'identification des micromammifères du Québec. Ministère de l'Environnement et de la Faune, Québec. 19 pages.

van Zyll de Jong, C. G. 1983. Traité des Mammifères du Canada, volume 1 : Les Marsupiaux et les Insectivores. Musée national des Sciences naturelles, Musées nationaux du Canada. Ottawa, Canada. 217 pages.

Reçu 28 octobre 2001

Acceptée 15 janvier 2005

\section{Gulls, Larus spp., Foraging at Pink Salmon, Oncorhynchus gorbuscha, Spawning Runs}

\section{MARY F. WILLSON}

5230 Terrace Place, Juneau, Alaska 99801 USA; e-mail mwillson@ gci.net

Willson, Mary F. 2004. Gulls, (Larus spp.), foraging at Pink Salmon, Oncorhynchus gorbuscha, spawning runs. Canadian Field-Naturalist 118(3): 442-443.

Small and immature gulls foraged more often on drifting salmon eggs than did large and mature gulls, and large and mature gulls foraged more often on salmon carcasses, at streams in Southeast Alaska. These differences may be related to body size via physical strength and dominance status, as well as foraging experience.

Key Words: Gulls, Larus spp., Pink Salmon, Oncorhynchus gorbuscha, Southeast Alaska.

Salmon spawning runs attract many foraging birds and mammals (Willson and Halupka 1995) and are clearly an important food resource for wildlife in late summer and fall. Gulls (Larus spp.) are among the most numerous predators and scavengers at salmon runs, sometimes occurring in the thousands (Isleib and Kessel 1973). Here I report how four species of gull exploited runs of Pink Salmon (Oncorhynchus gorbuscha) at the mouths of streams in Juneau, Alaska.

\section{Study Area and Methods}

Most of these observations were made at the mouths of Salmon Creek and Sheep Creek (less than $12 \mathrm{~km}$ apart), with some supplementary observations at four other small, nearby streams, within the city and borough of Juneau in Southeast Alaska (ca. $58^{\circ} 30^{\prime} \mathrm{N}$, $133^{\circ} 30^{\prime} \mathrm{W}$ ). I observed four species of gulls foraging at or near low tides, usually in the morning, from 27 August to 6 September 2002 ( $>6$ hrs of observation). At any one time, many dozens of gulls were present at the two primary study locations. At low tide, these creeks flow over intertidal deltas, often with several shallow channels used by incoming salmon. I scanned these deltas with spotting scope and binoculars, noting the foraging activities of the gulls and counting the individuals engaged in several distinctive foraging activities. Birds shifted position frequently, using different parts of the deltas; many individuals of the three larger species also changed foraging activity as I watched. Most scans were made at different locations on different days; if more than one scan occurred on the same day and stream delta, they were separated in time by about $30 \mathrm{~min}$. I recorded foraging observations in three categories: foraging on salmon eggs (drifting eggs are common because later spawners often disturb the gravels over the nests of earlier spawners, foot-paddling by gulls also stirs up poorly buried eggs), foraging on salmon carcasses (including some extraction of eggs from living or dead fish), and searching for invertebrates in Fucus mats (where amphipods and isopods were common) and tide pools. 